\title{
A Detection System with Controlled Surface Sensitivity for a New UHR SEM
}

Petr Sytar $\check{1}^{1}$, Jaroslav Jiruše ${ }^{1}$, Jan Páral ${ }^{1}$

1. TESCAN Brno s.r.o., Brno, Czech Republic

Control of the surface sensitivity of the detected signal electrons is beneficial for modern scanning electron microscopy. We present our initial results obtained on the new ultra-high resolution SEM with an extended detection system that allows the filtering of secondary electrons (SE), energy filtering of back-scattered electrons (BSE) and angular BSE selection. All three filtering possibilities lead to enhanced surface sensitivity. The tested SEM combines a high-potential tube with magnetic-electrostatic objective lens delivering ultra-high resolving power in the field-free mode and two-stage flexible scanning with extra wide field of view. It can also be combined with a focused ion beam column.

The highest surface-sensitive contrast can be obtained by imaging with secondary electrons. Two specially designed detectors between which the integral SE signal is divided can be used simultaneously. The axial SE signal is more surface-sensitive (see Fig. $1 \mathrm{a}$ and $\mathrm{b}$ ).

The energy filtration of BSE is a straightforward approach to control BSE surface sensitivity. It is already increased at lower energies due to low depth of BSE penetration into the sample [1]. High-pass BSE energy filtering may be used for even more enhanced surface sensitivity [2]. We designed the BSE detector incorporating a filtering grid that can be biased up to $6 \mathrm{kV}$ to yield low-loss BSE signal. The results are shown in Fig. $1 \mathrm{c}$ and d. The same principle can also be used to reduce charging artefacts.

At high acceleration voltages the energy-filtering grid would have too high a potential to be sufficiently insulated in practice. In these cases, angular BSE filtration may control the surface sensitivity. Topographic BSE contrast can be obtained with electrons generated at low take-off angles [3, 4]. Three BSE detectors are used (see Fig. 1 e-g). The narrow-angle BSE detector gives standard bulk BSE contrast, wide-angle BSE detector increases topography contrast from electrons with low initial take-off angles and axial BSE detector enhances surface sensitivity without topography.

\section{References:}

[1] L Reimer in "Scanning Electron Microscopy", ed. H K V Lotsch, (Springer Verlag, Berlin) p.142.

[2] H Jaksch, Proceedings of EMAS 2011 (2011), p. 255.

[3] T Luo and A Khursheed, Journal of Vacuum Science \& Technology B 25, (2007), p.2017.

[4] T Aoyama, M Nagoshib and K Satoc, Surface and Interface Analysis, 46, (2014), p.1291. 


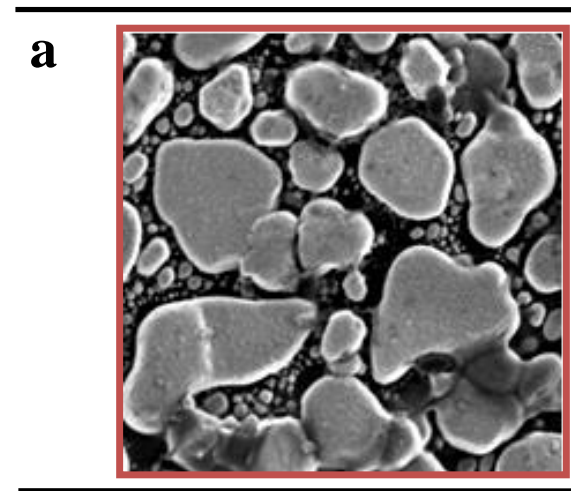

b
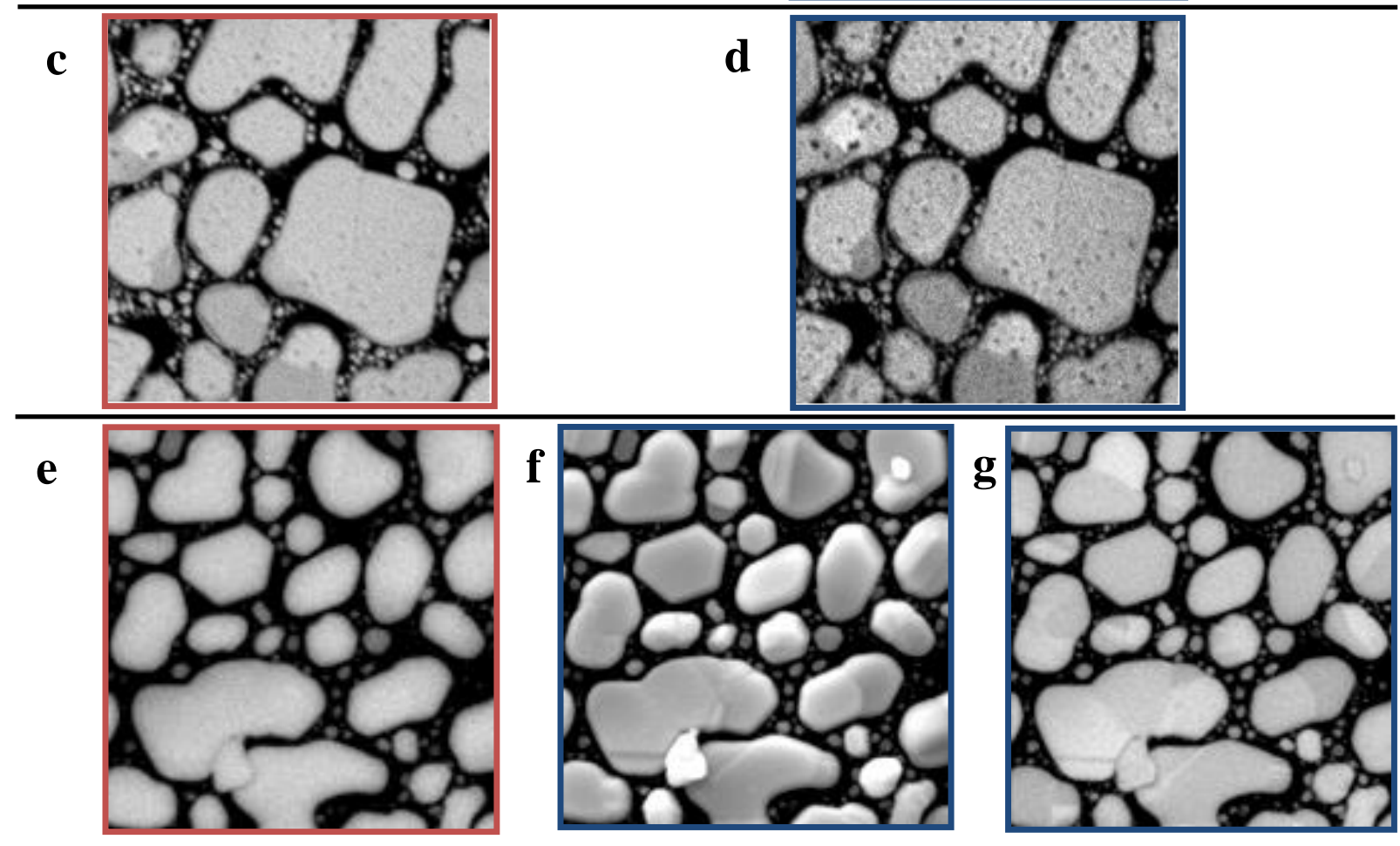

Figure 1. Controlled surface-sensitive imaging is shown on a sample of gold on carbon $(1.5 \mu \mathrm{m}$ field of view) at acceleration voltages of $500 \mathrm{~V}$ (a-b), $2 \mathrm{kV}$ (c-d) and $10 \mathrm{kV}$ (e-g). a) SE image of basic contrast and b) simultaneously acquired axial part of the SE signal with increased surface sensitivity. Energy selection: c) in-column BSE and d) increased surface sensitivity by detecting only in the 1800-2000 eV energy range. Angular selection: e) narrow-angle BSE image, f) wide-angle BSE with increased topography from electrons with low initial take-off angles and g) axial BSE with increased surface sensitivity without topography. 